\title{
The microbiota is a potential mediator of the crosstalk between $\gamma \delta$ T cells and tumors
}

\author{
Huidi Wang ${ }^{1,2}$, Jie $\mathrm{Li}^{2}$, Yan $\mathrm{He}^{2 *}$, Jia Yin ${ }^{1}{ }^{*}$ \\ ${ }^{1}$ Department of Neurology, Nanfang Hospital, Southern Medical University, Guangzhou 510080, Guangdong, China \\ ${ }^{2}$ Microbiome Medicine Center, Department of Laboratory Medicine, Zhujiang Hospital, Southern Medical University, Guangzhou \\ 510220, Guangdong, China
}

*Correspondence: Yan He, Microbiome Medicine Center, Department of Laboratory Medicine, Zhujiang Hospital, Southern Medical University, Guangzhou 510220, Guangdong, China. bioyanhe@gmail.com; Jia Yin, Department of Neurology, Nanfang Hospital, Southern Medical University, Guangzhou 510080, Guangdong, China. yinj@smu.edu.cn

Academic Editor: Dieter Kabelitz, Christian-Albrechts University of Kiel and University Hospital Schleswig-Holstein Campus Kiel, Germany

Received: October 21, 2021 Accepted: January 10, 2022 Published: February 18, 2022

Cite this article: Wang H, Li J, He Y, Yin J. The microbiota is a potential mediator of the crosstalk between $\gamma \delta$ T cells and tumors. Explor Immunol. 2022;2:48-63. https://doi.org/10.37349/ei.2022.00036

\begin{abstract}
$\gamma \delta \mathrm{T}$ cells are one of the immune cell types that express antigen receptors. $\gamma \delta \mathrm{T}$ cells are able to recognize pathogens or cancer cells independently of human leukocyte antigen restriction, which is an important feature of $\alpha \beta$ T cells. Therefore, $\gamma \delta$ T cells are considered the bridge between innate and adaptive immunity. These cells exhibit important roles in immune surveillance, exert immune defense against tumors and have become promising effector cells for cancer immunotherapy. However, in particular circumstances, the tumor microenvironment seems to render $\gamma \delta$ T cells immunosuppressive and even tumor-promoting, emphasizing the importance of regulating $\gamma \delta \mathrm{T}$ functions in realizing their translation into practical cancer immunotherapy. In recent years, increasing evidence has demonstrated that the intratumoral and peritumoral microbiota can have complex effects on tumor immunology. Thus, understanding the role of microbiota in the crosstalk between $\gamma \delta$ T cells and tumors will provide insights for developing adjuvant immunotherapy with precise regulation of tumor-related microbiota. In the present review, the effects of microbiota on $\gamma \delta$ T cell receptor repertoire and the roles of microbiota in some common tumors will be discussed, with implications for future cancer therapy.
\end{abstract}

\section{Keywords}

$\gamma \delta$ cells, tumor, microbiota, tumor micromilieu

\section{Introduction}

Discoveries in the field of $\gamma \delta \mathrm{T}$ cell biology have failed to keep the same pace as those in conventional $\alpha \beta \mathrm{T}$ cell research. Although $\gamma \delta$ T cells constitute only a minor subset of T lymphocytes, increased susceptibility to cancer development has been illustrated in $\gamma \delta \mathrm{T}$ cell deficient mice, suggesting an important role of $\gamma \delta \mathrm{T}$ cells in tumoral immunology [1]. Since the discovery of $\gamma \delta$ T cells in the 1980s, this unique component of the immune (C) The Author(s) 2022. This is an Open Access article licensed under a Creative Commons Attribution 4.0 International License (https://creativecommons.org/licenses/by/4.0/), which permits unrestricted use, sharing, adaptation, distribution and reproduction in any medium or format, for any purpose, even commercially, as long as you give appropriate credit to the original author(s) and the source, provide a link to the Creative Commons license, and indicate if changes were made. 
system has been considered a potentially new translational tool in cancer immunology and immunotherapy. Over the past few decades, researchers have unraveled the major differences of $\gamma \delta$ T cells from $\alpha \beta \mathrm{T}$ cells. $\gamma \delta \mathrm{T}$ cells (i) are derived from $\mathrm{CD}^{-} / \mathrm{CD}^{-}$double-negative thymocytes and mature earlier in the fetal thymus than $\alpha \beta$ T cells, (ii) are frequently associated with the epithelium, (iii) recognize multiple antigen-derived peptides with T cell receptors (TCRs) in a major histocompatibility complex (MHC)-independent manner, and (iv) present natural killer (NK) cell-like and antigen presenting cell (APC)-like innate reactivities. Therefore, $\gamma \delta \mathrm{T}$ cells possess a combination of innate and adaptive immune abilities. The broad and potent reactivities of $\gamma \delta$ $\mathrm{T}$ cells against tumors render them attractive candidates for immunotherapy. Many different methods have been investigated, including in vivo stimulation of target cells, adoptive infusion of autologous or allogeneic $\gamma \delta$ T cells after in vitro expansion, and so on.

The human body contains an estimated three trillion inhabitants that make up diverse, complex communities of microorganisms, referred to as microbiota. The microbiota, especially the gut microbiota, is known to extensively orchestrate various aspects of human physiology, from degrading food and extracting energy from indigestible carbohydrates and vitamin synthesis to modulating and stabilizing the nervous, metabolic and immune systems [2-5]. Although the number of microbiota inhabitants is matched to that of human cells in the body, the microbiota has a 100-fold higher genetic diversity than human cells, and the microbiota inhabitants influence not only their own bacterial niche but also host tissue-specific and immune functions [6]. It should be noted that gut microbiota profiles are highly dynamic and individualized, and are constantly shaped by internal and external stimuli. Moreover, researchers have also illustrated the variability and diversity of $\gamma \delta \mathrm{T}$ cell infiltration in different tumor entities [7].

All the above evidence emphasizes the need for a deep investigation of the role of tissue-resident microbiota in the interplay between tissue-specific $\gamma \delta$ T cells and tumors. In the present review, we discuss the factors that influence the $\gamma \delta$ TCR repertoires via microbiota. We focus our attention on some cancers with high morbidity and discuss how the microbiota may influence the interplay between $\gamma \delta \mathrm{T}$ cells and these cancers, with an eye toward future applications.

\section{TCR repertoire education by the microbiota}

To date, many clinical studies have investigated the efficacy of in vivo activation of autologous V $\gamma 9 \mathrm{~V} \delta 2 \mathrm{~T}$ cells (the dominate subset of $\gamma \delta$ T cells in peripheral blood of humans) by using aminobisphosphonates, and although no obvious harm has been reported, no substantial antitumoral activity has been observed [8-13]. Moreover, clinical studies that have investigated the ex vivo expansion of $\mathrm{V} \gamma 9 \mathrm{~V} \delta 2 \mathrm{~T}$ cells for the purpose of adoptive infusion have not been successful [14-23]. The failures of these clinical trials are most likely due to the limited understanding of $\gamma \delta$ TCR functional diversity and plasticity and the different manners of interaction between receptor and ligand.

During development, a $\gamma \delta$ T cell must go through the process of recombination of the T-cell receptor gamma $(T R G)$ and T-cell receptor delta $(T R D)$ gene loci to form a functional receptor. These TCR heterodimers consisting of one $\gamma$-chain $(T R G)$ and one $\delta$-chain (TRD) are assembled together by the combination of the variable $(V)$ gene segments, diversity $(D)$ gene segments and joining $(V)$ gene segments of the TRD. As such, each $\gamma \delta$ T cell presents a unique TCR that combines with CD3 on the cytomembrane of $\gamma \delta$ T cell to influence the signal transduction of the cell, which are important for the thymic selection and the induction of clonal cell proliferation stimulated by peripheral agents. As a result, the repertoire of $\gamma \delta$ TCRs is biased to overrepresent TCRs that can induce a specific signal to respond to the ligands. Although the ligands that bind to $\gamma \delta$ TCRs remains largely unknown, it is certain that these $\gamma \delta$ TCRs respond to a wide range of microbial-related products [24]. The varied and putative regions that bind to the ligand of $\gamma \delta$ TCRs are believed to be more similar to the immunoglobulin structure of antibodies than to $\alpha \beta$ TCRs [25].

Notably, V $\gamma 9 \mathrm{~V} \delta 2 \mathrm{~T}$ cells (which are the dominate $\gamma \delta \mathrm{T}$ cell subset in the blood of the second-trimester fetus) are functionally preprogrammed with properties of swiftly activatable innate-like $\mathrm{T}$ cells and expand during the first year of life, other than requiring bacterial exposure after birth [26]. This characteristic is believed to be a result of bacterial encounters and leads to the restricted development of an oligoclonal 
V $\gamma 9 \mathrm{~V} \delta 2$ TCR repertoire $[27,28]$. However, the $\mathrm{V} \gamma 9 \mathrm{~V} \delta 2 \mathrm{~T}$ cells still possess a large diversity, which leads them to have different functional avidities. It has been suggested that oligoclonal $\mathrm{V} \gamma 9 \mathrm{~V} \delta 2 \mathrm{~T}$ cells with a single but high-affinity TCR are functionally superior to cells that express a diverse V $\gamma 9 \mathrm{~V} \delta 2$ TCR [29]. Current $\gamma \delta \mathrm{T}$ cells-stimulating protocols that based on the broad and unselected stimulants tend to induce diverse specificities and avidities of the targeted cells. Consequently, it is very likely that high-quality $\gamma \delta \mathrm{T}$ cells with powerful function and specificity might be the ideal off-the-shelf cell products for adoptive transfer. Moreover, methods using products such as nitrogen-containing aminobisphosphonates to strongly activate $\gamma \delta$ TCR have been applied to expand $\mathrm{V} \gamma 9 \mathrm{~V} \delta 2 \mathrm{~T}$ cells in past clinical studies, but these strategies most likely resulted in the selection of low-affinity $\gamma \delta$ TCRs, therefore inducing limited tumor control. It is unclear what drives these clonal expansions and phenotypic development in the donors that seems to be healthy. Furthermore, a $\gamma \delta$ TCR-based activation strategy does not necessarily mobilize $\gamma \delta$ T cells with a NK receptor repertoire and Toll-like receptor (TLR) that help to identify multiple receptors on tumor cells. Therefore, selection of the right donor with optimally activated $\gamma \delta$ cells will definitely be of great importance in the design of $\gamma \delta \mathrm{T}$ cells immunotherapy.

In addition to the semi-invariant $\mathrm{V} \gamma 9 \mathrm{~V} \delta 2 \mathrm{~T}$ cells that are most abundant in the peripheral $\gamma \delta \mathrm{TCR}$ repertoire in most people, the rest of the subtypes are called non-V $\gamma 9 \mathrm{~V} \delta 2 \mathrm{~T}$ cells, which comprise a large diversity of different rearrangements of $\mathrm{V} \delta$ pairing with $\mathrm{V} \gamma$ and become different between individuals at some point after birth [30-32], which implies that external pathogenic stimuli are able to promote a vast oligoclonal expansion of non-V $\gamma 9 \mathrm{~V} \delta 2$ cells [27, 30, 31, 33]. As such, every individual has their own unique "fingerprint" in the form of the polyclonal TCR repertoire according to their history of encounters with different infectious agent. Within this context, current reports show that the failure of previous clinical trials expanding in vitro polyclonal $\gamma \delta$ T cells and transfer of the expanded cells into patients can be at least partially attributed to the high frequency of $\mathrm{V} \gamma 9 \mathrm{~V} \delta 2 \mathrm{~T}$ cells with poor tumor reactivities within healthy immune repertoires [34]. In a recent case report, researchers discovered that allogenic hematopoietic cell transplantation (HCT) in a patient with relapsing human papillomavirus (HPV) infections led to restoration of NK cell cytotoxicity, with persistent remission of all HPV-related diseases [35]. Interestingly, the patient presented different skin microbiota composition compared with the healthy controls and the microbiota dysbiosis was reversed after HCT. The success of a clinical trial by Xu et al. [23] begs the question of to what extent the microbiota profile of healthy donors is different from that of recipients.

An understanding of the importance of the commensal microbiota might provide insights into $\gamma \delta$ cell manipulation strategies. The immune system interacts with the microbiota inhabitants in various body sites. It has become more and more clear that bacterial antigens and their metabolic products participate in constant communication with the immune system, resulting in a microbiota-specific immune response. Though the uterus has long been traditionally thought to be sterile, researchers have reported the presence of commensal microbiota that resemble skin and oral microbiota in the amniotic fluid and placenta [36, 37]. In light of this finding, microbial education might begin in the uterus. In fact, the DNA of microbiota from the intestine could be found in the thymus of mice in early life [38]. At birth, newborn babies suddenly encounter various environmental cues that challenge their immune system. Their microbiota is acquired from the mother and the surrounding condition, and mutualistic crosstalk between the environment and the microbiota develop at all barrier sites. Adult $\gamma \delta$ T cells are phenotypically different from neonatal $\gamma \delta$ T cells in both TCR repertoire and innate- and adaptive-like functions $[39,40]$. The discrepancy in TCR $\delta$ repertoires between neonates and adults might result from different thymic output of $\gamma \delta$ T cells or extrathymic peripheral expansion of selective TCR repertoire responding to different bacterial antigens. A study conducted by Ravens et al. [41] suggested that $\gamma \delta$ TCR repertoires develop directly after birth and display significant differences among $\gamma \delta$ $\mathrm{T}$ cell subsets as a result of microbial exposure. Some individuals present private V $\gamma 9 \mathrm{~V} \delta 2$ expansions with phenotypically different functions, indicating selective expansion shaped by microbial stimulation [42]. Indeed, $\mathrm{V} \gamma 9 \mathrm{~V} \delta 2 \mathrm{~T}$ cells from cord blood present less response to pyrophosphate antigens than $\mathrm{V} \gamma 9 \mathrm{~V} \delta 2 \mathrm{~T}$ cells from adults $[43,44]$. Consistent with this finding, several types of infections expand $\mathrm{V} \gamma 9 \mathrm{~V} \delta 2 \mathrm{~T}$ cells, and strikingly, identical twins present different $\mathrm{V} \gamma 9 \mathrm{~V} \delta 2 \mathrm{~T}$ cell profiles [45]. 
Moreover, some subsets of $\gamma \delta \mathrm{T}$ cell repertoires are highly organ-specific, with $\mathrm{V} \gamma 4^{+}$cells located in the skin and liver, ${\mathrm{V} \gamma 5^{+}}^{+}$cells located in the skin epidermis, $\mathrm{V} \gamma 6^{+}$cells located in the urogenital tract and lungs, and $\mathrm{V} \gamma 7^{+}$intraepithelial lymphocytes (IELs) located in the gut [46]. In terms of TCR $\delta \mathrm{T}$ cells, V $\delta 1 \mathrm{~T}$ cells are mainly located in the skin and mucous membranes and incorporate $V \gamma 2, \gamma 3, \gamma 4, \gamma 5$ and $\gamma 8$ chains to maintain stability of the epithelium [47]. Commensal microbiota colonized many parts of the body, including the gastrointestinal tract, skin, respiratory tract, oral cavity, and urogenital tract. The compositions of microbiota are highly varied between different sites of the body (Figure 1). Moreover, different individuals harbor radically different collections of microbiota in the same body site, and little is known about what leads to this variation and what regulates it [6]. Recently, a review by Papotto et al. [48] dissected the role of microbiota in $\gamma \delta \mathrm{T}$ cell development and function and evaluated up-to-date evidence for $\gamma \delta \mathrm{T}$ cell selection in commensal microbiota.

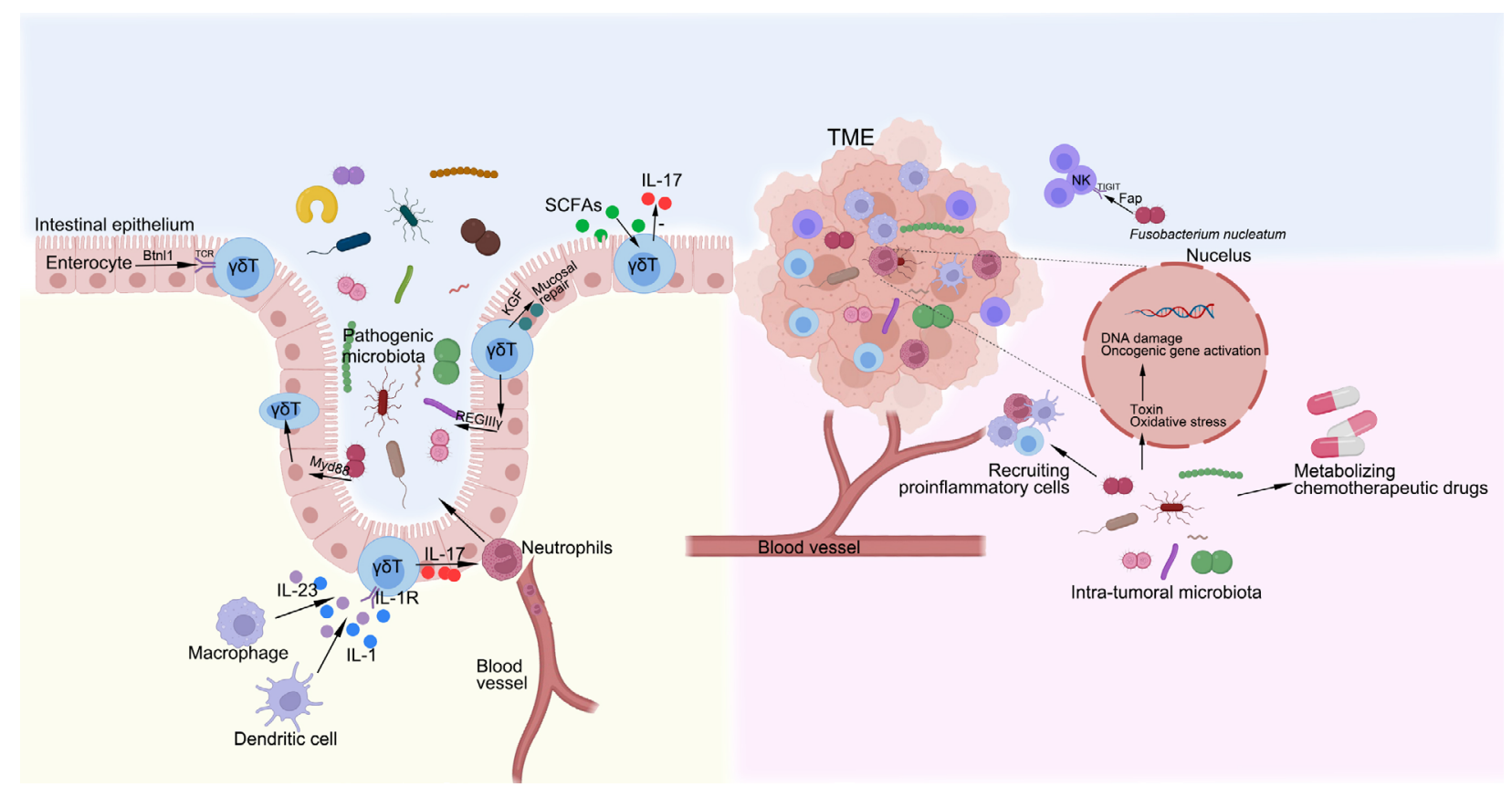

Figure 1. The interactions between microbiota, $ү \delta T$ cells and tumor. The development of $\gamma \delta$ IELs is depend on epithelial butyrophilin-like 1 (Btnl1) in a TCR-dependent manner, uninfluenced by the gut microbiota. In contrast, the dynamic and metabolic status of $y \delta$ IELs depend on pathogen sensing by enterocytes via Myd88 pathway. A population of $y \delta$ T cells, particularly cells that express interleukin-1 (IL-1) receptor 1, are activated by the macrophages and dendritic cells via gut microbiota to produce IL-17, which mediates a protective response to the intestinal pathogens by recruiting neutrophils from the blood vessel. In response to epithelial MyD88 signaling, $\gamma \delta$ IELs produce the antimicrobial peptide REGIIly, which regulates microbiota. The $y \delta$ IELs can also produce keratinocyte growth factor (KGF) that aids in mucosal repair. Gut microbiota-derived short-chain fatty acids (SCFAs) reduce IL-17 production by manipulating $\mathrm{\gamma} \delta \mathrm{T}$ cells. The mechanisms underlying carcinogenic effects of intra-tumoral microbiota, mainly include producing toxin and oxidative stress to damage DNA, activating expression of oncogenic genes, selectively recruiting proinflammatory cells and metabolizing chemotherapeutic drugs. Notably, Fusobacterium nucleatum, a microbiota that is found within the colon adenocarcinoma, can specifically target the inhibitory receptor T-cell immunoglobulin and immunoreceptor tyrosine-based inhibitory motif domains (TIGIT), via its Fap2 protein, to downregulate the activities of NK cells. TME: tumor microenvironment

Although most $\gamma \delta \mathrm{T}$ cells studies have focused on $\mathrm{V} \gamma 9 \mathrm{~V} \delta 2 \mathrm{~T}$ cells, $\mathrm{V} \delta 1$ cells are attracting increasing interest. Studies show that V $\delta 1 \mathrm{~T}$ cells express higher levels of cytotoxicity markers and induce greater lysis of colorectal cancer (CRC) cell lines than V $\delta 2 \mathrm{~T}$ cells $[49,50]$.

It has long been observed by clinical physicians that acute infection can be positively related to spontaneous cancer regression, and it is currently well appreciated that the potent immune response against acute infection can overcome malignancy [51,52]. Using two different lung cancer models, researchers found that microbiota-based stimuli that mimicked an acute infection led to a remarkable reduction in tumor burden [53]. Interestingly, Kalyan et al. [54] found that subcutaneously injected microbial immunomodulators derived from several inactivated strains had antitumor effects on some specific organs. This result gave rise to an exciting new perspective that organ-specific stimulation of the antitumor activity of $\gamma \delta \mathrm{T}$ cells can be activated by microbiota that are endogenously located in specific organs. Although the therapeutic 
potential of trained innate immunity of $\gamma \delta \mathrm{T}$ cells has yet to be fully realized, it is possible that microbial immunomodulators would provide important additional assistance in a tissue-specific manner for $\gamma \delta \mathrm{T}$ cells, a concept that deserves further investigation. On the other hand, $\gamma \delta \mathrm{T}$ cells can also exert protumor effects. These effects are mainly because of $\gamma \delta$ T17 cells that produce IL-17. Currently, accumulating evidence supporting the notion that tissue-resident $V \delta 1 \mathrm{~T}$ cells tend to differentiate into $\gamma \delta \mathrm{T} 17$ cells in many tumors, including CRC [55], lung cancer [56] and breast cancer [57].

\section{The interactions between microbiota, $\gamma \delta$ T cells and tumor}

The bidirectional interactions between the microbiota and $\gamma \delta \mathrm{T}$ cells influence both homeostasis and pathologies of the body. It has been shown that in the intestinal mucosa, the development of $\gamma \delta$ IELs is depend on epithelial Btnl1 in a TCR-dependent manner, uninfluenced by the gut microbiota [58]. In contrast, another study [59] using high-resolution microscope and intersectional genetic instruments demonstrates that $\gamma \delta$ IELs exhibit unique microbiota-dependent location and movement patterns in the epithelial compartment. The dynamic and metabolic status of $\gamma \delta$ IELs depend on pathogen sensing by enterocytes [59]. A population of CD62L- $\gamma \delta$ T cells, particularly cells that express IL-1 receptor 1 , are activated by the macrophages and dendritic cells via gut microbiota to produce IL-17 [60]. Moreover, the $\gamma \delta$ T17 cells in the lamina propria are dependent on the microbiota, possibly via indirect process, since it has been demonstrated that IL-10 secreted by forkhead box protein $3\left(\right.$ Foxp $\left.^{+}\right)$regular $\mathrm{T}$ (Treg) in the lamina propria suppresses expansion of $\gamma \delta$ T17 cells in the stroke context [61], and Foxp $3^{+}$Treg are increased in germ-free mice while $\gamma \delta$ T17 cells are decreased [62]. On the other hand, $\gamma \delta$ IELs are able to promote expression of regenerating islet-derived III gamma (RegIII $\gamma$ ), an antimicrobial peptide, which constrain the growth of bacteria when intestinal mucosal damage occurs [63]. In turn, the immunomodulatory and antibacterial properties of $\gamma \delta$ T cells are affected by the gut microbiota. Notably, epithelial cells are able to sense the gut microbiota via MyD88-dependent manner and control the metabolism of $\gamma \delta$ T cells and epithelial surveillance [59]. $\gamma \delta$ IELs contribute to mucosal repair via the production of KGF [64]. In the skin, $\gamma \delta$ T17 cells interact with Corynebacterium in a mycolic acid/IL-23-dependent manner [65].

In the context of tumorigenesis, the ability of microbiota in activating anti-tumor potential of $\gamma \delta \mathrm{T}$ cells represents a promising prospect. One possible link lies in the microbial metabolites. Indeed, estrogen produced by the gut microbiota can enter the circulation, thus affecting the risk of sex hormone-driven cancers. Moreover, gut microbiota-derived SCFAs and secondary bile acids can affect cancer progression [66]. SCFA is one of the most studied microbial metabolites that take part in the cancer immunity, mainly by counteracting the effect of histone deacetylase and thereby affects the differentiation and activation of anti-inflammatory Treg cells or pro-inflammatory T helper 1 (Th1) and Th17 cells, polarization of macrophages [67]. Recently, a study conducted by Dupraz et al. [68] demonstrates that SCFAs, particularly propionate, reduce IL-17 production by manipulating $\gamma \delta$ T cells in mice and humans with inflammatory bowel disease. Further studies on whether SCFAs participate in the regulation of $\gamma \delta \mathrm{T}$ cells are warranted.

With the advance of metagenomic sequencing technique, the intra-tumoral microbiota has been identified as a component of the TME, affecting the tumor development and therapeutic effect. The mechanisms underlying carcinogenic effects of intra-tumoral microbiota, mainly include producing toxin and oxidative stress to damage DNA, activating expression of oncogenic genes, selectively recruiting proinflammatory cells and metabolizing chemotherapeutic drugs [69]. For instance, Fusobacterium nucleatum can generate a proinflammatory TME that promotes colorectal neoplasia progression by recruiting tumor-infiltrating immune cells [70].

Hypoxia is a common feature of solid tumors, the hypoxic core of the tumor creates a microenvironment for the growth and accumulation of anaerobic microbiota. Interestingly, a study conducted by Li et al. [71] shows that oxygen pressure in TME orchestrates the expansion and cytotoxicity of $\gamma \delta \mathrm{T}$ cell via tumor-derived exosomes. Nevertheless, small-size tumors or metastatic tumors have a higher oxygen, so facultative anaerobes such as Salmonella and Escherichia are more adapted. Some hypoxic tumors can recruit bacteria to accumulate in the TME via some small molecules concurrently with Listeria spp. infection 
and consist in myeloid-derived suppressor cells (MDSCs) to avoid immune clearance [72]. Notably, the TME is an unstable environment with an enhanced immune suppression, which helps to evade immune recognition. The intra-tumoral microbiota may play an important role in this evasion. For example, Fusobacterium nucleatum, a microbiota that is found within the colon adenocarcinoma, can specifically target the inhibitory receptor TIGIT, via its Fap2 protein, to downregulate the activities of NK cells [73].

\section{The role of microbiota in the interplay between $\gamma \delta$ T cells and lung cancer}

Lung cancer is a malignant tumor and has the highest mortality and morbidity worldwide [74]. The oncogenesis of lung cancer is closely related to chronic inflammation. However, the causes and immune mediators of this inflammation remain elusive. The lung is a mucosal organ harboring many microbial communities and is exposed to a variety of airborne microbiota and environmental particles through inhalation. Lung-resident microbiota are closely associated with certain lung diseases, including lung cancer, because microbiota dysbiosis might increase the risk of cancer by influencing metabolism and the immune response, increasing inflammation, and promoting toxicity [75]. Thus, understanding the composition of microbiota in lung cancer patients is conducive to exploring the mechanism of lung cancer.

The TME, comprising tumor cells, the microvasculature, tumor-associated fibroblasts, immune cells and extracellular components, influences the therapeutic efficacy of immunotherapy. $\gamma \delta \mathrm{T}$ cells have been found to infiltrate tumors where they exert immunological effects. The clinical relevance of $\gamma \delta$ T cells in the TME is not clear because of conflicting results [76]. Different studies using different methods, such as flow cytometry or immunohistochemistry, to detect tumor-infiltrating $\gamma \delta$ T cells have reported an association of $\gamma \delta$ T cells with tumor progression or tumor regression or even a lack of correlation with prognosis [76]. Recent advances in sequencing technology have led to the discovery and comprehensive characterization of microbiota within tumors, linking microbiota with the TME. TMEs are known to possess complexity, heterogeneity and substantial individual differences [77]. It is worth noting that neoantigens exhibit homology with infectious disease-derived peptides, implying that a neoantigen might mimic microbial epitopes through neoantigen molecular mimicry [78]. In recent years, a large amount of evidence has demonstrated that intratumoral microbiota can have a complex impact on the TME via different immune pathways [79-81]. In a murine model of pancreatic ductal adenocarcinoma, researchers detected specific gut-derived microbiota inhabitants in tumors, suggesting potential microbial translocation from the gastrointestinal tract to the peritumoral milieu; such translocation led to innate and adaptive immune suppression and tumor progression [82].

A study using bronchoalveolar lavage fluid samples showed that the phyla Firmicutes and TM7 and the genera Veillonella and Megasphaera were increased in lung cancer patients [83]. However, the biological effects of specific microbiota inhabitants on the tumorigenesis of lung cancer are unclear. Pulmonary homeostasis is maintained by lung-resident immune cells, which provide immune surveillance in response to invading hazardous pathogens [84]. Jin et al. [56] observed a difference in the abundance of lung-resident $\gamma \delta \mathrm{T}$ cells between healthy specific pathogen-free and germ-free mice, indicating that the presence of a commensal microbiota is indispensable in maintaining tissue-resident $\gamma \delta$ T cells in homeostatic circumstances. They also discovered that commensal bacteria contribute to inflammation and tumorigenesis by stimulating IL- $1 \beta$ and IL-23 expressions from myeloid cells in a Myd88-dependent manner, which further promote proliferation of IL-17-producing $\mathrm{V} \gamma 6^{+} \mathrm{V} \delta 1^{+} \mathrm{T}$ cells.

More importantly, researchers found that elimination of the microbiota or inhibition of $\gamma \delta$ T cells in the lung cancer model did not lead to any change in the immune response of $\alpha \beta \mathrm{T}$ cells. This finding is in line with another study, which showed that $\gamma \delta$ T cells support pancreatic oncogenesis by restraining $\alpha \beta \mathrm{T}$ cells [85]. The commensal microbiota in the lung indirectly stimulates IL-17 expression in lung-resident $\gamma \delta 1 \mathrm{~T}$ cells to promote inflammation and tumor development. In fact, IL-17 has been proven to promote tumorigenesis via several effects, including upregulation of angiogenesis, adhesion molecule expression and endothelial permeability [86]. An opposing pattern was observed by Cheng et al. [87], who found that a commensal microbiota was essential for the function of $\gamma \delta \mathrm{T} 17$ cells in the lung. The abrogation of this microbiota by antibiotics hampered the secretion of IL-17 and increased the susceptibility to tumor development, suggesting 
a highly context-dependent microbiota-immune cell interaction. Shi et al. [88] discovered a close positive correlation between $\gamma \delta$ T cells and the $\alpha$-diversity of the microbiota in the lungs of cancer patients. However, studies of the lung microbiota and the interplay between the lung-resident microbiota and pulmonary immunity are limited, and additional mechanistic insights remain to be exploited in the future.

\section{The role of microbiota in the interplay between $\gamma \delta$ T cells and CRC}

CRC is the 3rd most-common malignant tumor in the world [89]. The constituents of the microbiota in the small intestine are very different from those in the colon. The bacterial load is much higher in the colon than in the small intestine, partly because of the acidic environment, which is inhospitable for bacteria [90]. Therefore, it is likely that immunity in the small intestine is different from that in the colon. Accordingly, human cancer mainly occurs in the colon and scarcely occurs in the small intestine. Studies have provided compelling evidence supporting the causal role of the gut microbiota in the development of CRC [91-93]. The microbiota in the tumor is very different from that in the adjacent healthy tissue [94]. Additionally, evidence from some preclinical studies has suggested gut microbiota dysbiosis as an oncogenic driver of CRC, and some specific bacterial species can promote tumorigenesis by inducing the release of proinflammatory toxins (such as Bacteroides fragilis) or reactive oxygen species (Fusobacterium nucleatum) [70, 95]. To date, most studies have mainly focused on the role of cytotoxic T cells in CRC, while an unbiased, comprehensive investigation of both innate and adaptive immune cells in CRC is lacking. Using high-dimensional mass cytometry, flow cytometry and single cell RNA sequencing, de Vries et al. [96] provided an unbiased characterization of the immune component of CRC. They discovered that a previously unappreciated innate lymphocyte population was enriched in tumor tissues and presented cytotoxic activity. Two populations of $\gamma \delta$ T cells were identified: the programmed cell death protein 1 (PD-1)+ population was almost exclusively present in DNA mismatch repair (MMR)-deficient tumors, and the PD-1- counterpart population, was found in normal mucosa and MMR-proficient tumors. $\gamma \delta$ T cells are the major innate immune cell population in the intestinal epithelium that are involved in the crosstalk between the gut microbiota and CRC. V $\delta 1 \mathrm{~T}$ cells originate from the thymus and reside in the colonic epithelium, are the major IELs. Physiological development and antimicrobial ability acquisition of IELs depend on constant education by the gut microbiota. Additionally, the absence of $\gamma \delta \mathrm{T}$ cells leads to enhanced formation of colorectal adenocarcinoma and $\gamma \delta$ T cells reportedly present antitumor activity in the context of CRC in humans [97, 98].

However, the roles of $\gamma \delta$ T cells in CRC have only begun to be uncovered. For example, although $\gamma \delta \mathrm{T}$ cells (mainly $\mathrm{V} \gamma 9 \mathrm{~V} \delta 2 \mathrm{~T}$ cells) have been employed in a series of clinical trials, including in vivo activation and adoptive transfer of in vitro-expanded cells, the clinical responses to treatment are far from satisfactory $[20,99]$. This lack of effect may be a result of the complexity of the TME of CRC. In CRC initiation, circulating V $\gamma 9 \mathrm{~V} \delta 2 \mathrm{~T}$ cells converge in tumor sites, displaying Th1 cell-like properties and killing tumor cells. In contrast, as the tumor progress, MDSCs and tumor-associated macrophages become enriched in the TME; here, they secrete IL-1 $\beta$, IL-23 and transforming growth factor- $\beta$ (TGF- $\beta$ ), which reprogrammes recruited $\mathrm{V} \gamma 9 \mathrm{~V} \delta 2 \mathrm{~T}$ cells into $\gamma \delta \mathrm{T} 17$ cells or regulatory $\gamma \delta \mathrm{T}$ cells, leading to an immune-suppressive TME [100]. To improve future $\gamma \delta$ T cell immunotherapies, the role of the gut microbiota in the TME needs to be investigated. Whether $\gamma \delta \mathrm{T}$ cells can be reprogrammed by precise modulation of the microbiota composition to transform the TME towards an antitumor state will need to be determined.

A classic example of a certain bacteria that has been confirmed as a key precursor to carcinogens is Proteobacterium Helicobacter pylori, which colonizes the stomach mucous membrane and contributes to carcinogenesis of gastric cancer through chronic inflammation [101]. Specifically, H. pylori has been reported to activate nucleotide-binding oligomerization domain-containing protein 1 (NOD-1)-like receptor, which stimulates an IL-33 dependent response, resulting in excessive generation of inflammatory macrophages and T cell phenotypes [102]. An in vitro experiment demonstrates that $H$. pylori can directly activate $\mathrm{CD}^{+}{ }^{+} \mathrm{T}$ cells especially $\gamma \delta$ T cells with TCR chain $V \delta 2$, therefore creating an inflammatory environment which favors the chronic persistence of $H$. pylori and contributes to the gastric cancer [103]. 


\section{The role of microbiota in the interplay between $\gamma \delta$ T cells and oral mucosa}

The oral mucosa is constantly exposed to microbes, antigens, allergens and endure frequent microdamage due to masticatory movement, making it a site susceptible to chronic inflammatory disease such as periodontitis. Therefore, in order to withstand a wide range of bacterial insults and mechanical challenges, the oral mucosa consist of a tissue-specific immune cells, in which $\gamma \delta$ T cells are critical mediators. It has been reported that the Tcrd-GDL mouse, a model of $\gamma \delta$ T cells ablation, presents elevated gingival inflammation, with increased neutrophils and monocytes and Foxp $3^{+}$cells, i.e., Tregs [104]. In another study, ablation of $\gamma \delta \mathrm{T}$ cells results in decreased expression of growth arrest-specific protein 6 (GAS6), an important regulator of oral mucosal homeostasis in oral mucosa, and levels of IL-17 were upregulated and led to an overall increased IL-17/Foxp3 ratio, indicating an increased local inflammation [105]. Moreover, $\gamma \delta$ T cells are important in maintaining oral mucosal integrity, as they produce the wound healing associated cytokine amphiregulin, which helps repair oral pathology of mice [106].

The specific localization and gradual development of oral $\gamma \delta$ T cells imply that they are educated by the resident microbiota. Indeed, the ratio of gingival ${\mathrm{V} \gamma 6^{+}}^{+}$and $\mathrm{V} \gamma 4^{+}$cells along with level of IL-17, a cytokine expressed by $\mathrm{V} \gamma 6^{+}$subset, are reduced in germ-free mice and on the other hand, conditional ablation of $\gamma \delta$ $\mathrm{T}$ cells leads to exacerbation of gingival inflammation and altered oral microbiota diversity [104]. Of note, the oral microbiota has a profound impact on the development of mucosal Langerhans cells, which maintain mucosal homeostasis. The adult germ-free mice present a significant reduction of $50 \%$ in the frequency of Langerhans cells in the gingival epithelium compared to specific pathogen-free mice [107]. In addition, a significant microbiota dysbiosis, featured by an overgrowth of anaerobic bacteria by utilizing inflammatory byproducts for anaerobic respiration, was observed in GAS6 deficient mice [105]. The most likely mechanism underling microbial upregulation of $\gamma \delta \mathrm{T} 17$ cells is through activating pattern recognition receptors expressed by dendritic cells and direct activation by microbiota via TLR $[108,109]$.

Oral squamous cell carcinoma (OSCC) accounts for approximately $90 \%$ of oral malignancies and $2 \%$ of all cancers around the globe [74]. OSCC is associated with several oral microbiota, e.g., Fusobacterium nucleatum, Porphyromonas gingivalis and Streptococcus sp. [110]. The mechanisms underlying the involvement of oral microbiota in the carcinogenesis of oral cancer may include induction of chronic inflammation, direct contact with cells and regulation of local immunity. As $\gamma \delta$ T cells make up a large proportion of immune cells of the oral squamous epithelium, they ought to be among the first line of defense to early carcinogenic transformation. As a matter of fact, a higher proportion of $\gamma \delta$ T cells was observed in patients with OSCC, which was not paralleled with apoptotic tumor cells [111]. Since $\gamma \delta$ T17 cells are the majority of oral $\gamma \delta \mathrm{T}$ cells, it is likely that these cells may exert deleterious effects on the tumorigenesis of OSCC. Future studies are needed to address this important question.

\section{The role of microbiota in gynecological cancer}

The female reproductive tract (FRT), like other mucosal layers, is colonized by a diverse yet special microbiota inhabitants, which are important in maintaining homeostasis. Anatomically, the FRT consist of two parts, the lower FRT (vagina and cervix) and the upper FRT (uterus, fallopian tubes and ovaries), which display different microbiota composition. The most abundant microbiota in the FRT is Lactobacillus, which mainly resides in the vagina. However, owing to the low microbial biomass, our knowledge about the microbiota that reside in the upper FRT is still in its infancy and is a challenging topic to be uncovered [112]. Predominant vaginal Lactobacillus spp. are beneficial to the microenvironment, protecting it from invading pathogens by inducing microbial products and preventing pathogenic adhesions [80]. Importantly, the lactic acid that acidifies the vaginal microenvironment and leads to a $\mathrm{pH}$ level lower than 4.5 , is a major mechanism of protection in lower FRT. Indeed, numerous studies have shown that lactic acid can protect FRT from a wide range of sexually transmitted pathogens [80]. Lactobacillus reduction, which is a sign of FRT dysbiosis, has been associated with many gynecological diseases, including cancer. Cervical cancer is the most common HPV-associated cancer and accounts for 2 nd highest death rates for female cancer [74]. Over the past decade, more and more evidence imply that the vaginal microbiota may participant in the tumorigenesis of cervical cancer. Individuals 
with non-Lactobacillus-dominant vaginal microbiota are at a higher risk of HPV infection and persistence [80]. Of note, a significant increase in vaginal $\mathrm{pH}$ is observed to be associated with the severity of neoplasia and strongly correlated with the reduction of Lactobacillus [113]. To date, published studies investigating the mechanism underlying the interactions between vaginal microbiota and HPV, which is difficult to cultivate in vitro, are limited. Furthermore, mouse model for bacterial vaginosis [114] or HPV-induced cervical dysplasia and cancer are limited [115]. However, future studies utilizing these animal model will gain insights into the role of vaginal microbiota in the tumorigenesis of cervical cancer with $\gamma \delta$ T cells participation.

\section{Conclusions}

Overall, $\gamma \delta$ T cell development is shaped by the resident microbiota both prenatally and postnatally, and this process has a great impact on the TCR repertoire and TCR reactivity. Moreover, the intra-tumoral microbiota may play an important role in modulating the TME. These issues warrant further investigation, and clearly, the role of the microbiota in the crosstalk between $\gamma \delta \mathrm{T}$ cells and tumor cells has been underappreciated. Undoubtedly, to optimally utilize the great potential of $\gamma \delta$ T cells in immunotherapies, we must significantly improve our knowledge and understanding of their extensive diversity, plasticity, and multifunctionality as well as how these cells can be harnessed both precisely and safely in therapeutic applications.

\section{Abbreviations}

CRC: colorectal cancer

Foxp3: forkhead box protein 3

FRT: female reproductive tract

HPV: human papillomavirus

IELs: intraepithelial lymphocytes

IL-1: interleukin-1

NK: natural killer

OSCC: oral squamous cell carcinoma

SCFAs: short-chain fatty acids

TCRs: T cell receptors

Th1: T helper 1

TME: tumor microenvironment

$T R D$ : T-cell receptor delta

Treg: regular $\mathrm{T}$

\section{Declarations}

Author contributions

HW and JL wrote the manuscript, YH and JY conceived and revised the manuscript.

\section{Conflicts of interest}

The authors declare that they have no conflicts of interest.

Ethical approval

Not applicable.

\section{Consent to participate}

Not applicable. 


\section{Consent to publication}

Not applicable.

Availability of data and materials

Not applicable.

\section{Funding}

Not applicable.

\section{Copyright}

(C) The Author(s) 2022.

\section{References}

1. Girardi M, Oppenheim DE, Steele CR, Lewis JM, Glusac E, Filler R, et al. Regulation of cutaneous malignancy by $\gamma \delta$ T cells. Science. 2001;294:605-9.

2. Zheng D, Liwinski T, Elinav E. Interaction between microbiota and immunity in health and disease. Cell Res. 2020;30:492-506.

3. Sharon G, Sampson TR, Geschwind DH, Mazmanian SK. The central nervous system and the gut microbiome. Cell. 2016;167:915-32.

4. Rastelli M, Cani PD, Knauf C. The gut microbiome influences host endocrine functions. Endocr Rev. 2019;40:1271-84.

5. Cani PD. Human gut microbiome: hopes, threats and promises. Gut. 2018;67:1716-25.

6. Gilbert JA, Blaser MJ, Caporaso JG, Jansson JK, Lynch SV, Knight R. Current understanding of the human microbiome. Nat Med. 2018;24:392-400.

7. Chabab G, Boissiere-Michot F, Mollevi C, Ramos J, Lopez-Crapez E, Colombo PE, et al. Diversity of tumor-infiltrating, $\gamma \delta$ T-cell abundance in solid cancers. Cells. 2020;9:1537.

8. Wilhelm M, Kunzmann V, Eckstein S, Reimer P, Weissinger F, Ruediger T, et al. $\gamma \delta \mathrm{T}$ cells for immune therapy of patients with lymphoid malignancies. Blood. 2003;102:200-6.

9. Dieli F, Vermijlen D, Fulfaro F, Caccamo N, Meraviglia S, Cicero G, et al. Targeting human $\gamma \delta$ T cells with zoledronate and interleukin-2 for immunotherapy of hormone-refractory prostate cancer. Cancer Res. 2007;67:7450-7.

10. Poccia F, Gioia C, Martini F, Sacchi A, Piacentini P, Tempestilli M, et al. Zoledronic acid and interleukin-2 treatment improves immunocompetence in HIV-infected persons by activating $\mathrm{V} \gamma 9 \mathrm{~V} \delta 2 \mathrm{~T}$ cells. AIDS. 2009;23:555-65.

11. Bennouna J, Levy V, Sicard H, Senellart H, Audrain M, Hiret S, et al. Phase I study of bromohydrin pyrophosphate (BrHPP, $\mathrm{IPH}_{1101}$ ), a V $\gamma 9 \mathrm{~V} \delta 2 \mathrm{~T}$ lymphocyte agonist in patients with solid tumors. Cancer Immunol Immunother. 2010;59:1521-30.

12. Meraviglia S, Eberl M, Vermijlen D, Todaro M, Buccheri S, Cicero G, et al. In vivo manipulation of V $\gamma 9 \mathrm{~V} \delta 2$ $\mathrm{T}$ cells with zoledronate and low-dose interleukin-2 for immunotherapy of advanced breast cancer patients. Clin Exp Immunol. 2010;161:290-7.

13. Kunzmann V, Smetak M, Kimmel B, Weigang-Koehler K, Goebeler M, Birkmann J, et al. Tumor-promoting versus tumor-antagonizing roles of $\gamma \delta$ T cells in cancer immunotherapy: results from a prospective phase I/II trial. J Immunother. 2012;35:205-13.

14. Kobayashi H, Tanaka Y, Yagi J, Osaka Y, Nakazawa H, Uchiyama T, et al. Safety profile and anti-tumor effects of adoptive immunotherapy using gamma-delta T cells against advanced renal cell carcinoma: a pilot study. Cancer Immunol Immunother. 2007;56:469-76. 
15. Bennouna J, Bompas E, Neidhardt EM, Rolland F, Philip I, Galea C, et al. Phase-I study of Innacell $\gamma \delta^{\mathrm{TM}}$, an autologous cell-therapy product highly enriched in $\gamma 9 \delta 2 \mathrm{~T}$ lymphocytes, in combination with IL-2, in patients with metastatic renal cell carcinoma. Cancer Immunol Immunother. 2008;57:1599-609.

16. Abe Y, Muto M, Nieda M, Nakagawa Y, Nicol A, Kaneko T, et al. Clinical and immunological evaluation of zoledronate-activated $V \gamma 9 \gamma \delta$ T-cell-based immunotherapy for patients with multiple myeloma. Exp Hematol. 2009;37:956-68.

17. Nakajima J, Murakawa T, Fukami T, Goto S, Kaneko T, Yoshida Y, et al. A phase I study of adoptive immunotherapy for recurrent non-small-cell lung cancer patients with autologous $\gamma \delta$ T cells. Eur J Cardiothorac Surg. 2010;37:1191-7.

18. Kobayashi H, Tanaka Y, Yagi J, Minato N, Tanabe K. Phase I/II study of adoptive transfer of $\gamma \delta$ T cells in combination with zoledronic acid and IL-2 to patients with advanced renal cell carcinoma. Cancer Immunol Immunother. 2011;60:1075-84.

19. Nicol AJ, Tokuyama H, Mattarollo SR, Hagi T, Suzuki K, Yokokawa K, et al. Clinical evaluation of autologous gamma delta T cell-based immunotherapy for metastatic solid tumours. Br J Cancer. 2011;105:778-86.

20. Izumi T, Kondo M, Takahashi T, Fujieda N, Kondo A, Tamura N, et al. Ex vivo characterization of $\gamma \delta$ T-cell repertoire in patients after adoptive transfer of $\mathrm{V} \gamma 9 \mathrm{~V} \delta 2 \mathrm{~T}$ cells expressing the interleukin-2 receptor $\beta$-chain and the common $\gamma$-chain. Cytotherapy. 2013;15:481-91.

21. Wada I, Matsushita H, Noji S, Mori K, Yamashita H, Nomura S, et al. Intraperitoneal injection of in vitro expanded $\mathrm{V} \gamma 9 \mathrm{~V} \delta 2 \mathrm{~T}$ cells together with zoledronate for the treatment of malignant ascites due to gastric cancer. Cancer Med. 2014;3:362-75.

22. Aoki T, Matsushita H, Hoshikawa M, Hasegawa K, Kokudo N, Kakimi K. Adjuvant combination therapy with gemcitabine and autologous $\gamma \delta$ T-cell transfer in patients with curatively resected pancreatic cancer. Cytotherapy. 2017;19:473-85.

23. Xu Y, Xiang Z, Alnaggar M, Kouakanou L, Li J, He J, et al. Allogeneic V $\gamma 9 V \delta 2$ T-cell immunotherapy exhibits promising clinical safety and prolongs the survival of patients with late-stage lung or liver cancer. Cell Mol Immunol. 2021;18:427-39.

24. Girardi M. Immunosurveillance and immunoregulation by $\gamma \delta$ T cells. J Invest Dermatol. 2006;126:25-31.

25. Rock EP, Sibbald PR, Davis MM, Chien YH. CDR3 length in antigen-specific immune receptors. J Exp Med. 1994;179:323-8.

26. Dimova T, Brouwer M, Gosselin F, Tassignon J, Leo O, Donner C, et al. Effector V $\gamma 9 \mathrm{~V} \delta 2 \mathrm{~T}$ cells dominate the human fetal $\gamma \delta$ T-cell repertoire. Proc Natl Acad Sci U S A. 2015;112:E556-65.

27. Davey MS, Willcox CR, Hunter S, Kasatskaya SA, Remmerswaal EBM, Salim M, et al. The human V $\delta 2^{+}$ T-cell compartment comprises distinct innate-like V $\delta 9^{+}$and adaptive V $\delta 9^{-}$subsets. Nat Commun. 2018;9:1760.

28. Fisch P, Malkovsky M, Kovats S, Sturm E, Braakman E, Klein BS, et al. Recognition by human $\mathrm{V}_{\gamma} 9 / \mathrm{V}_{\delta} 2 \mathrm{~T}$ cells of a GroEL homolog on Daudi Burkitt's lymphoma cells. Science. 1990;250:1269-73.

29. Grunder C, van Dorp S, Hol S, Drent E, Straetemans T, Heijhuurs S, et al. $\gamma 9$ and $\delta 2 C D R 3$ domains regulate functional avidity of T cells harboring $\gamma 9 \delta 2 T C R s$. Blood. 2012;120:5153-62.

30. Ravens S, Schultze-Florey C, Raha S, Sandrock I, Drenker M, Oberdorfer L, et al. Human $\gamma \delta$ T cells are quickly reconstituted after stem-cell transplantation and show adaptive clonal expansion in response to viral infection. Nat Immunol. 2017;18:393-401. Erratum in: Nat Immunol. 2018;19:1037.

31. Davey MS, Willcox CR, Joyce SP, Ladell K, Kasatskaya SA, McLaren JE, et al. Clonal selection in the human V $\delta 1 \mathrm{~T}$ cell repertoire indicates $\gamma \delta$ TCR-dependent adaptive immune surveillance. Nat Commun. 2017;8:14760.

32. Pitard V, Roumanes D, Lafarge X, Couzi L, Garrigue I, Lafon ME, et al. Long-term expansion of effector/ memory $V \delta 2^{-} \gamma \delta$ T cells is a specific blood signature of CMV infection. Blood. 2008;112:1317-24. 
33. Kallemeijn MJ, Kavelaars FG, van der Klift MY, Wolvers-Tettero ILM, Valk PJM, van Dongen JJM, et al. Next-generation sequencing analysis of the human $\operatorname{TCR} \gamma \delta^{+}$T-cell repertoire reveals shifts in $V^{-}$and V $\delta$-usage in memory populations upon aging. Front Immunol. 2018;9:448.

34. Sebestyen Z, Prinz I, Dechanet-Merville J, Silva-Santos B, Kuball J. Translating gammadelta $(\gamma \delta)$ T cells and their receptors into cancer cell therapies. Nat Rev Drug Discov. 2020;19:169-84.

35. Lisco A, Hsu AP, Dimitrova D, Proctor DM, Mace EM, Ye P, et al. Treatment of relapsing HPV diseases by restored function of natural killer cells. N Engl J Med. 2021;385:921-9.

36. Aagaard K, Ma J, Antony KM, Ganu R, Petrosino J, Versalovic J. The placenta harbors a unique microbiome. Sci Transl Med. 2014;6:237ra65.

37. Collado MC, Rautava S, Aakko J, Isolauri E, Salminen S. Human gut colonisation may be initiated in utero by distinct microbial communities in the placenta and amniotic fluid. Sci Rep. 2016;6:23129.

38. Zegarra-Ruiz DF, Kim DV, Norwood K, Kim M, Wu WH, Saldana-Morales FB, et al. Thymic development of gut-microbiota-specific T cells. Nature. 2021;594:413-7.

39. Hayday AC. $\gamma \delta \mathrm{T}$ cell update: adaptate orchestrators of immune surveillance. J Immunol. 2019;203:311-20.

40. Vermijlen D, Prinz I. Ontogeny of innate T lymphocytes-some innate lymphocytes are more innate than others. Front Immunol. 2014;5:486.

41. Ravens S, Fichtner AS, Willers M, Torkornoo D, Pirr S, Schöning J, et al. Microbial exposure drives polyclonal expansion of innate $\gamma \delta$ T cells immediately after birth. Proc Natl Acad Sci U S A. 2020;117:18649-60.

42. Willcox CR, Davey MS, Willcox BE. Development and selection of the human V $\gamma 9 \mathrm{~V} \delta 2^{+} \mathrm{T}$-cell repertoire. Front Immunol. 2018;9:1501.

43. Cairo C, Sagnia B, Cappelli G, Colizzi V, Leke RG, Leke RJ, et al. Human cord blood $\gamma \delta$ T cells expressing public $\mathrm{V} \gamma_{2}$ chains dominate the response to bisphosphonate plus interleukin-15. Immunology. 2013;138:346-60.

44. Tomchuck SL, Leung WH, Dallas MH. Enhanced cytotoxic function of natural killer and $\mathrm{CD}^{+}{ }^{+} \mathrm{CD} 56^{+}$cells in cord blood after culture. Biol Blood Marrow Transplant. 2015;21:39-49.

45. Parker CM, Groh V, Band H, Porcelli SA, Morita C, Fabbi M, et al. Evidence for extrathymic changes in the T cell receptor gamma/delta repertoire. J Exp Med. 1990;171:1597-612.

46. Park JH, Lee HK. Function of $\gamma \delta \mathrm{T}$ cells in tumor immunology and their application to cancer therapy. Exp Mol Med. 2021;53:318-27.

47. Vantourout P, Hayday A. Six-of-the-best: unique contributions of $\gamma \delta \mathrm{T}$ cells to immunology. Nat Rev Immunol. 2013;13:88-100.

48. Papotto PH, Yilmaz B, Silva-Santos B. Crosstalk between $\gamma \delta$ T cells and the microbiota. Nat Microbiol. 2021;6:1110-7.

49. Halary F, Pitard V, Dlubek D, Krzysiek R, de la Salle H, Merville P, et al. Shared reactivity of V $\delta 2^{\text {neg }}$ $\gamma \delta \mathrm{T}$ cells against cytomegalovirus-infected cells and tumor intestinal epithelial cells. J Exp Med. 2005;201:1567-78.

50. Wu D, Wu P, Wu X, Ye J, Wang Z, Zhao S, et al. Ex vivo expanded human circulating V $\delta 1 \gamma \delta \mathrm{T}$ cells exhibit favorable therapeutic potential for colon cancer. Oncoimmunology. 2015;4:e992749.

51. Jessy T. Immunity over inability: the spontaneous regression of cancer. J Nat Sci Biol Med. 2011;2:43-9.

52. Thomas JA, Badini M. The role of innate immunity in spontaneous regression of cancer. Indian J Cancer. 2011;48:246-51.

53. Bazett M, Costa AM, Bosiljcic M, Anderson RM, Alexander MP, Wong SWY, et al. Harnessing innate lung anti-cancer effector functions with a novel bacterial-derived immunotherapy. Oncoimmunology. 2018; 7:e1398875. 
54. Kalyan S, Bazett M, Sham HP, Bosiljcic M, Luk B, Dhanji S, et al. Distinct inactivated bacterial-based immune modulators vary in their therapeutic efficacies for treating disease based on the organ site of pathology. Sci Rep. 2020;10:5901.

55. Wu P, Wu D, Ni C, Ye J, Chen W, Hu G, et al. $\gamma \delta T 17$ cells promote the accumulation and expansion of myeloid-derived suppressor cells in human colorectal cancer. Immunity. 2014;40:785-800.

56. Jin C, Lagoudas GK, Zhao C, Bullman S, Bhutkar A, Hu B, et al. Commensal microbiota promote lung cancer development via $\gamma \delta$ T cells. Cell. 2019;176:998-1013.e16.

57. Ma C, Zhang $Q$, Ye J, Wang F, Zhang Y, Wevers E, et al. Tumor-infiltrating $\gamma \delta$ T lymphocytes predict clinical outcome in human breast cancer. J Immunol. 2012;189:5029-36.

58. Di Marco Barros R, Roberts NA, Dart RJ, Vantourout P, Jandke A, Nussbaumer O, et al. Epithelia use butyrophilin-like molecules to shape organ-specific $\gamma \delta$ T cell compartments. Cell. 2016;167:203-18.e17.

59. Hoytema van Konijnenburg DP, Reis BS, Pedicord VA, Farache J, Victora GD, Mucida D. Intestinal epithelial and intraepithelial $\mathrm{T}$ cell crosstalk mediates a dynamic response to infection. Cell. 2017;171:783-94.e13.

60. Duan J, Chung H, Troy E, Kasper DL. Microbial colonization drives expansion of IL-1 receptor 1-expressing and IL-17-producing $\gamma / \delta$ T cells. Cell Host Microbe. 2010;7:140-50.

61. Benakis C, Brea D, Caballero S, Faraco G, Moore J, Murphy M, et al. Commensal microbiota affects ischemic stroke outcome by regulating intestinal $\gamma \delta$ T cells. Nat Med. 2016;22:516-23.

62. Ivanov, II, Frutos Rde L, Manel N, Yoshinaga K, Rifkin DB, Sartor RB, et al. Specific microbiota direct the differentiation of IL-17-producing T-helper cells in the mucosa of the small intestine. Cell Host Microbe. 2008;4:337-49.

63. Ismail AS, Behrendt CL, Hooper LV. Reciprocal interactions between commensal bacteria and $\gamma \delta$ intraepithelial lymphocytes during mucosal injury. J Immunol. 2009;182:3047-54.

64. Chen Y, Chou K, Fuchs E, Havran WL, Boismenu R. Protection of the intestinal mucosa by intraepithelial $\gamma \delta$ T cells. Proc Natl Acad Sci U S A. 2002;99:14338-43.

65. St Leger AJ, Desai JV, Drummond RA, Kugadas A, Almaghrabi F, Silver P, et al. An ocular commensal protects against corneal infection by driving an interleukin-17 response from mucosal $\gamma \delta \mathrm{T}$ Cells. Immunity. 2017;47:148-58.e5.

66. Cheng P, Shen P, Shan Y, Yang Y, Deng R, Chen W, et al. Gut microbiota-mediated modulation of cancer progression and therapy efficacy. Front Cell Dev Biol. 2021;9:626045.

67. Zhang Z, Tang H, Chen P, Xie H, Tao Y. Demystifying the manipulation of host immunity, metabolism, and extraintestinal tumors by the gut microbiome. Signal Transduct Target Ther. 2019;4:41.

68. Dupraz L, Magniez A, Rolhion N, Richard ML, Da Costa G, Touch S, et al. Gut microbiota-derived short-chain fatty acids regulate IL-17 production by mouse and human intestinal $\gamma \delta \mathrm{T}$ cells. Cell Rep. 2021;36:109332.

69. An Y, Zhang W, Liu T, Wang B, Cao H. The intratumoural microbiota in cancer: new insights from inside. Biochim Biophys Acta Rev Cancer. 2021;1876:188626.

70. Kostic AD, Chun E, Robertson L, Glickman JN, Gallini CA, Michaud M, et al. Fusobacterium nucleatum potentiates intestinal tumorigenesis and modulates the tumor-immune microenvironment. Cell Host Microbe. 2013;14:207-15.

71. Li L, Cao B, Liang X, Lu S, Luo H, Wang Z, et al. Microenvironmental oxygen pressure orchestrates an anti- and pro-tumoral $\gamma \delta$ T cell equilibrium via tumor-derived exosomes. Oncogene. 2019;38:2830-43.

72. Quispe-Tintaya W, Chandra D, Jahangir A, Harris M, Casadevall A, Dadachova E, et al. Nontoxic radioactive Listeri $^{\text {at }}$ is a highly effective therapy against metastatic pancreatic cancer. Proc Natl Acad Sci U S A. 2013;110:8668-73. 
73. Gur C, Ibrahim Y, Isaacson B, Yamin R, Abed J, Gamliel M, et al. Binding of the Fap2 protein of Fusobacterium nucleatum to human inhibitory receptor TIGIT protects tumors from immune cell attack. Immunity. 2015;42:344-55.

74. Sung H, Ferlay J, Siegel RL, Laversanne M, Soerjomataram I, Jemal A, et al. Global cancer statistics 2020: GLOBOCAN estimates of incidence and mortality worldwide for 36 cancers in 185 countries. CA Cancer J Clin. 2021;71:209-49.

75. Mao Q Jiang F, Yin R, Wang J, Xia W, Dong G, et al. Interplay between the lung microbiome and lung cancer. Cancer Lett. 2018;415:40-8.

76. Silva-Santos B, Serre K, Norell H. $\gamma \delta$ T cells in cancer. Nat Rev Immunol. 2015;15:683-91.

77. Klein CA. Cancer progression and the invisible phase of metastatic colonization. Nat Rev Cancer. 2020;20:681-94.

78. Balachandran VP, Łuksza M, Zhao JN, Makarov V, Moral JA, Remark R, et al. Identification of unique neoantigen qualities in long-term survivors of pancreatic cancer. Nature. 2017;551:512-6.

79. Helmink BA, Khan MAW, Hermann A, Gopalakrishnan V, Wargo JA. The microbiome, cancer, and cancer therapy. Nat Med. 2019;25:377-88.

80. Łaniewski P, Ilhan ZE, Herbst-Kralovetz MM. The microbiome and gynaecological cancer development, prevention and therapy. Nat Rev Urol. 2020;17:232-50.

81. Sánchez-Alcoholado L, Ramos-Molina B, Otero A, Laborda-Illanes A, Ordóñez R, Medina JA, et al. The role of the gut microbiome in colorectal cancer development and therapy response. Cancers (Basel). 2020;12:1406.

82. Pushalkar S, Hundeyin M, Daley D, Zambirinis CP, Kurz E, Mishra A, et al. The pancreatic cancer microbiome promotes oncogenesis by induction of innate and adaptive immune suppression. Cancer Discov. 2018;8:403-16.

83. Lee SH, Sung JY, Yong D, Chun J, Kim SY, Song JH, et al. Characterization of microbiome in bronchoalveolar lavage fluid of patients with lung cancer comparing with benign mass like lesions. Lung Cancer. 2016;102:89-95.

84. Lloyd CM, Marsland BJ. Lung homeostasis: influence of age, microbes, and the immune system. Immunity. 2017;46:549-61.

85. Daley D, Zambirinis CP, Seifert L, Akkad N, Mohan N, Werba G, et al. $\gamma \delta$ T cells support pancreatic oncogenesis by restraining $\alpha \beta$ T cell activation. Cell. 2016;166:1485-99.e15.

86. Silva-Santos B, Mensurado S, Coffelt SB. $\gamma \delta$ T cells: pleiotropic immune effectors with therapeutic potential in cancer. Nat Rev Cancer. 2019;19:392-404.

87. Cheng M, Qian L, Shen G, Bian G, Xu T, Xu W, et al. Microbiota modulate tumoral immune surveillance in lung through a $\gamma \delta \mathrm{T} 17$ immune cell-dependent mechanism. Cancer Res. 2014;74:4030-41.

88. Shi R, Li Y, Liu H, Chen J. Lung microbiota: unexploited treasure hidden in the immune microenvironment of lung cancer. Thorac Cancer. 2021;12:2964-6.

89. Siegel RL, Miller KD, Fedewa SA, Ahnen DJ, Meester RGS, Barzi A, et al. Colorectal cancer statistics, 2017. CA Cancer J Clin. 2017;67:177-93.

90. Berg RD. The indigenous gastrointestinal microflora. Trends Microbiol. 1996;4:430-5.

91. Sears CL, Garrett WS. Microbes, microbiota, and colon cancer. Cell Host Microbe. 2014;15:317-28.

92. Yang Y, Jobin C. Novel insights into microbiome in colitis and colorectal cancer. Curr Opin Gastroenterol. 2017;33:422-7.

93. Brennan CA, Garrett WS. Gut microbiota, inflammation, and colorectal cancer. Annu Rev Microbiol. 2016;70:395-411.

94. Lu Y, Chen J, Zheng J, Hu G, Wang J, Huang C, et al. Mucosal adherent bacterial dysbiosis in patients with colorectal adenomas. Sci Rep. 2016;6:26337. 
95. Purcell RV, Pearson J, Aitchison A, Dixon L, Frizelle FA, Keenan JI. Colonization with enterotoxigenic Bacteroides fragilis is associated with early-stage colorectal neoplasia. PLoS One. 2017;12:e0171602.

96. de Vries NL, van Unen V, Ijsselsteijn ME, Abdelaal T, van der Breggen R, Farina Sarasqueta A, et al. High-dimensional cytometric analysis of colorectal cancer reveals novel mediators of antitumour immunity. Gut. 2020;69:691-703.

97. Matsuda S, Kudoh S, Katayama S. Enhanced formation of azoxymethane-induced colorectal adenocarcinoma in $\gamma \delta$ T lymphocyte-deficient mice. Jpn J Cancer Res. 2001;92:880-5.

98. Mikulak J, Oriolo F, Bruni E, Roberto A, Colombo FS, Villa A, et al. NKp46-expressing human gut-resident intraepithelial V $\delta 1 \mathrm{~T}$ cell subpopulation exhibits high antitumor activity against colorectal cancer. JCI Insight. 2019;4:e125884.

99. Noguchi A, Kaneko T, Kamigaki T, Fujimoto K, Ozawa M, Saito M, et al. Zoledronate-activated V $\gamma 9 \gamma \delta \mathrm{T}$ cell-based immunotherapy is feasible and restores the impairment of $\gamma \delta \mathrm{T}$ cells in patients with solid tumors. Cytotherapy. 2011;13:92-7.

100. Yang $\mathrm{Y}, \mathrm{Xu} \mathrm{C}, \mathrm{Wu} \mathrm{D}$, Wang Z, Wu P, Li L, et al. $\gamma \delta$ T cells: crosstalk between microbiota, chronic inflammation, and colorectal cancer. Front Immunol. 2018;9:1483.

101. Wang F, Meng W, Wang B, Qiao L. Helicobacter pylori-induced gastric inflammation and gastric cancer. Cancer Lett. 2014;345:196-202.

102. Tran LS, Tran D, De Paoli A, D'Costa K, Creed SJ, Ng GZ, et al. NOD1 is required for Helicobacter pylori induction of IL-33 responses in gastric epithelial cells. Cell Microbiol. 2018;20:e12826.

103. Romi B, Soldaini E, Pancotto L, Castellino F, Del Giudice G, Schiavetti F. Helicobacter pylori induces activation of human peripheral $\gamma \delta^{+}$T lymphocytes. PLoS One. 2011;6:e19324.

104. Wilharm A, Tabib Y, Nassar M, Reinhardt A, Mizraji G, Sandrock I, et al. Mutual interplay between IL-17-producing $\gamma \delta$ T cells and microbiota orchestrates oral mucosal homeostasis. Proc Natl Acad Sci U S A. 2019;116:2652-61.

105. Nassar M, Tabib Y, Capucha T, Mizraji G, Nir T, Pevsner-Fischer M, et al. GAS6 is a key homeostatic immunological regulator of host-commensal interactions in the oral mucosa. Proc Natl Acad Sci U S A. 2017;114:E337-46.

106. Krishnan S, Prise IE, Wemyss K, Schenck LP, Bridgeman HM, McClure FA, et al. Amphiregulin-producing $\gamma \delta \mathrm{T}$ cells are vital for safeguarding oral barrier immune homeostasis. Proc Natl Acad Sci U S A. 2018;115:10738-43.

107. Capucha T, Koren N, Nassar M, Heyman O, Nir T, Levy M, et al. Sequential BMP7/TGF- $\beta 1$ signaling and microbiota instruct mucosal Langerhans cell differentiation. J Exp Med. 2018;215:481-500.

108. Ness-Schwickerath KJ, Morita CT. Regulation and function of IL-17A- and IL-22-producing $\gamma \delta$ T cells. Cell Mol Life Sci. 2011;68:2371-90.

109. Martin B, Hirota K, Cua DJ, Stockinger B, Veldhoen M. Interleukin-17-producing $\gamma \delta$ T cells selectively expand in response to pathogen products and environmental signals. Immunity. 2009;31:321-30.

110. Stasiewicz M, Karpiński TM. The oral microbiota and its role in carcinogenesis. Semin Cancer Biol. 2021; [Epub ahead of print].

111. Bas M, Bier H, Schirlau K, Friebe-Hoffmann U, Scheckenbach K, Balz V, et al. Gamma-delta T-cells in patients with squamous cell carcinoma of the head and neck. Oral Oncol. 2006;42:691-7.

112. Baker JM, Chase DM, Herbst-Kralovetz MM. Uterine microbiota: residents, tourists, or invaders? Front Immunol. 2018;9:208.

113. Łaniewski P, Barnes D, Goulder A, Cui H, Roe DJ, Chase DM, et al. Linking cervicovaginal immune signatures, HPV and microbiota composition in cervical carcinogenesis in non-Hispanic and Hispanic women. Sci Rep. 2018;8:7593. 
114. Herbst-Kralovetz MM, Pyles RB, Ratner AJ, Sycuro LK, Mitchell C. New systems for studying intercellular interactions in bacterial vaginosis. J Infect Dis. 2016;214 Suppl 1:6-13.

115. Christensen ND, Budgeon LR, Cladel NM, Hu J. Recent advances in preclinical model systems for papillomaviruses. Virus Res. 2017;231:108-18. 